\title{
Differentiated Thyroid Cancer: Assessment of Clinical Practice in a Tertiary Referral Centre
}

\author{
P.W. Owens ${ }^{1}$, A.J. Lowery ${ }^{1}$, M. Bell ${ }^{2}$, D.S. Quill ${ }^{1}$, M.J. Kerin ${ }^{1}$
}

1. Department of Surgery, University Hospital Galway, Newcastle Road, Galway, Ireland

2. Department of Endocrinology, University Hospital Galway, Newcastle Road, Galway, Ireland

\section{Introduction}

Thyroid cancer is Ireland's most common endocrine malignancy, accounting for $1 \%$ of cancers with an incidence of 162 cases per year. ${ }^{1}$ International best practice guidelines issued by the British Thyroid Association (BTA 2014) ${ }^{2}$ provide well-defined recommendations for the management of differentiated thyroid cancer (DTC) measuring $\leq 1 \mathrm{~cm}$ and $>4 \mathrm{~cm}$, and for those with defined risk factors. However, the extent of surgery and requirement for radioiodine remnant ablation (RRA) are less clearly defined for tumours measuring $1-4 \mathrm{~cm}$ in size. Guidelines recommend a "personalised decision making" approach for this cohort, with therapeutic decisions based on patient preference, recurrence risk and multi-disciplinary discussion.

\section{Aims}

- Describe patterns of DTC and its management in an Irish tertiary referral centre.

- Assess adherence to current practice guidelines (BTA).

- Investigate management strategies in the "Personalised decision making" cohort.

\section{Nethods}

Patients treated for DTC in a tertiary referral centre between 2009 and 2014 were included. Data was obtained from a prospectively maintained thyroid cancer database. Patient demographics and disease parameters including tumour characteristics (histology/TNM staging parameters), surgical intervention and the use of RRA were recorded.

\section{Results}

97 patients treated for DTC between 2009 and 2014 were assessed. 68 (70\%) were female. Mean age was 47 (+/- SD 15 years). 85 (88\%) exhibited papillary histology while the remaining $12 \%$ were follicular tumours. Mean tumour size was $31 \mathrm{~mm}$. Observed treatment patterns are as depicted in Figure 1.

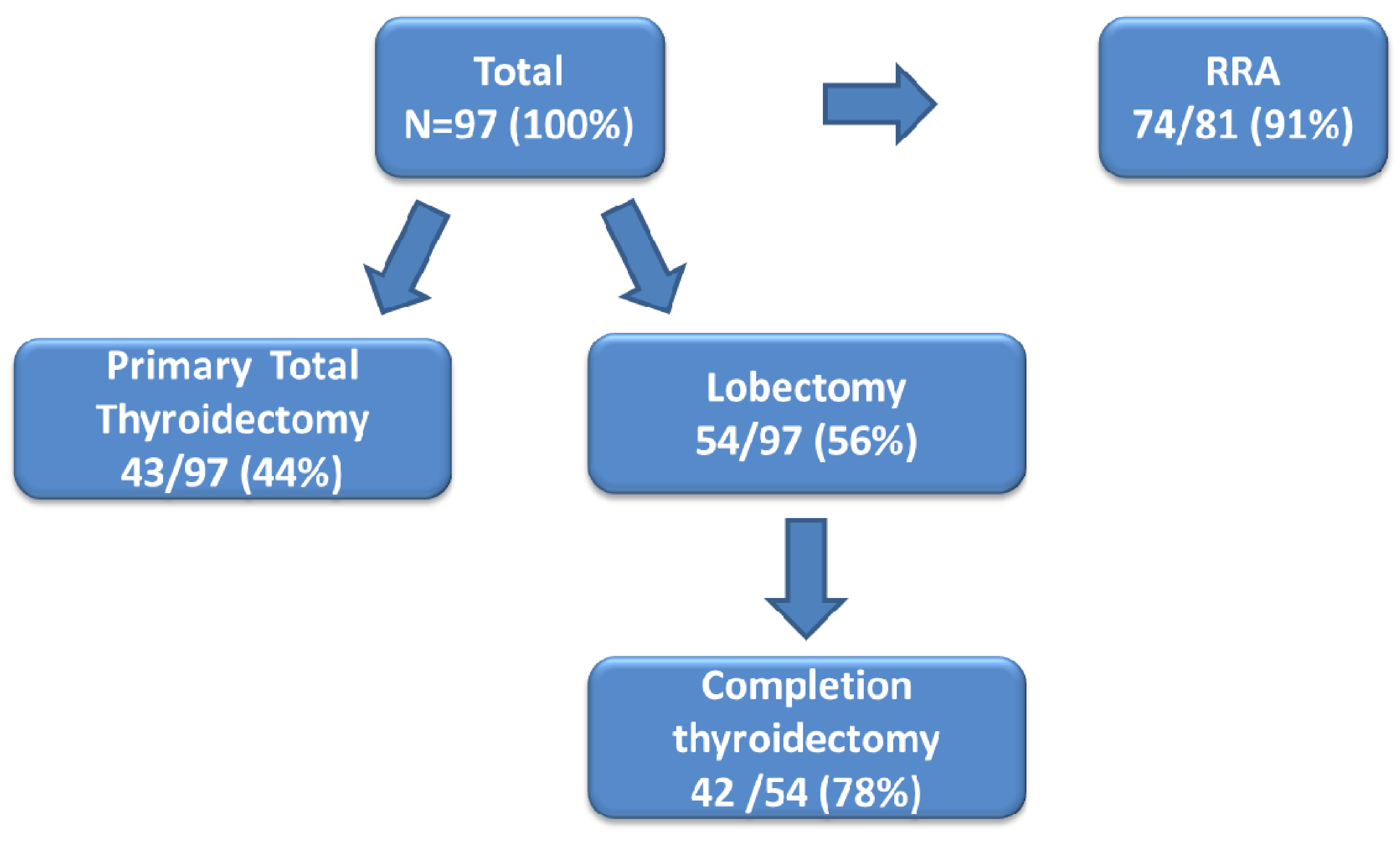

Figure 1 - Surgical and RRA Treatment Patterns

\section{Results}

94 out of 97 patients (97\%) were managed in accordance with guidelines (Table 1). Out of those whose management did not adhere to guidelines, three were over treated with total thyroidectomy, while two were under treated with lobectomy only. 28 patients $(29 \%)$ had tumour characteristics requiring "personalised decision making", of which 26 underwent total thyroidectomy rather than lobectomy. Similarly for RRA, 39 out of 41 patients (95\%) in the "personalised decision making" group were treated with radioactive iodine.

\begin{tabular}{|ccc|}
\hline & Patients (\%) & $\begin{array}{c}\text { Adherence to } \\
\text { Surgical Guidelines } \\
(\%)\end{array}$ \\
\hline Total Cohort: & $97(100 \%)$ & $94(97 \%)$ \\
\hline Tumour Size $\leq 1 \mathrm{~cm}:$ & $17(18 \%)$ & $16(94 \%)$ \\
\hline Tumour Size 1-4cm: & $61(63 \%)$ & $60(98 \%)$ \\
\hline Tumour Size >4cm: & $19(20 \%)$ & $18(95 \%)$ \\
\hline Multifocal: & $40(41 \%)$ & $39(98 \%)$ \\
\hline Lymphovascular Invasion: & $21(22 \%)$ & $21(100 \%)$ \\
\hline Capsular Invasion: & $21(22 \%)$ & $21(100 \%)$ \\
\hline Age > 45 & $53(55 \%)$ & $46(87 \%)$ \\
\hline
\end{tabular}

Table 1 - Tumour Characteristics and Adherence to BTA Guidelines

All 11 patients (100\%) in the surgical "Personalised decision making" cohort underwent the more aggressive treatment in the form of total thyroidectomy, while 43 out of $45(96 \%)$ in the RRA "Personalised Decision group" went on to receive radioiodine ablation. All cases were discussed at endocrinology multidisciplinary meetings attended by surgeons, endocrinologists, radiologists and pathologists. The median tumour size for this cohort was $24 \mathrm{~mm}(+/-$ SD $9 \mathrm{~mm}$ ) and $96 \%$ of these tumours exhibited papillary histology. As expected, none were multifocal, extracapsular, node positive or exhibited lymphovascular invasion.

\section{Conclusions}

- Management of this cohort exhibited high levels of adherence to internationally recognised best practice guidelines.

- A tendency towards more aggressive surgical and RRA intervention was observed in the "Personalised decision making" group.

- Good quality prospective trials are required to provide adequate evidence for those patients who fall into the "Personalised decision making" treatment groups.

\section{References}

1. Perros, P. et al. (2014). "Guidelines for the Management of Thyroid Cancer." J Clinical Endocrinology (Oxf) 81 Supplement 1: 1-122.

2. National Cancer Registry (2014). "Cancer in Ireland 1994-2011: Annual report of the National Cancer Registry." 